\title{
Satellite Image Compression Using ROI Based EZW Algorithm
}

\author{
A. Vinay ${ }^{*}{ }^{1}$, S. Natarajan ${ }^{2}$ \\ ${ }^{1}$ Information Technology, AMET University, Chennai, India \\ ${ }^{2}$ Department of computer science, PES institute of Technology, Bangalore, India
}

\begin{abstract}
In all the fields that make use of the images in a large scale for the image applications there is need for the image compression process in order to minimize the size of the storage. Likewise in the marine field there is use of the images like satellite images for their communication purpose. So to make use of them we are proposing a new image compression technique for the compression of the satellite images by using the Region of Interest (ROI)based on the lossy image technique called the Embedded Zero-Tree Wavelet (EZW) algorithm for the compression. The performance of our method can be evaluated and analyzing the PSNR values of the output images.
\end{abstract}

Keywords: PSO,NB,J48, cache replacement algorithms, AWRP, dynamic aging

\section{Introduction}

In all the fields that make use of the images in a large scale for the image applications there is need for the image compression process in order to minimize the size of the storage. Likewise in the marine field there is use of the images like satellite images for their communication purpose.

\subsection{Background}

Region of interest coding techniques for medical image compression is described in [1]. $\mathrm{ROI}$ coding is to permit the use of arbitrarily and multiple shaped ROls with random weights describing the degree of significance for every ROI images. Medical image compression based on hybrid DWT with Back Propagation Neural Network (BPNN) approach is described in [2]. Compressed image quality is improved by DWT technique and BP algorithm can be widely used as a learning algorithm. The paper discussed in [3] uses the hybrid algorithm for the image compression technique. The hybrid algorithm is DWT for achieving a higher compression ratio and the DCT with the Huffman algorithm is used to preserve the quality of the reconstructed image. An adaptive sampling algorithm is used in [4], for evaluating the area difference between the predicted and the correct points to decide the significant coefficients. A method to increase the compression ratio with less computational burden is discussed in [5]. In order to decomposition of a sub-block into equal sized bands, the DCT is used as a band pass filter and a high similarity property is found among the bands. Region of interest (ROI) based on compression of image is discussed in [6] for classification applications. The method is a fusion of both lossy and lossless compression along with wavelets transforms like Embedded ZeroTree Wavelet (EZW) is as derived techniques. An image compression method based on Integer Wavelet Transform (IWT) and SVD is discussed in [7]. A graph based quantization is used in the method but the adaptive Huffman coding is used for entropy encoding. An improved SPIRT algorithm in which most of the energy is intense in the low frequency sub-band after wavelet transform is discussed in [8]. To compress the information that are sent form one place to another by means of the low frequency coefficients the LZW algorithm is used in Image Super Resolution Using Wavelet Transformation Based Genetic Algorithm [9]. This compression algorithm is done for the ROI based extracted image. The statistics analysis associated with difference image is discussed in image super resolution reconstruction using iterative adaptive regularization method and genetic algorithm [10]. The paper is based on the statistical analysis measure when compared with the compression of the lossless image. The Security of Arithmetic Compression Based Text Steganography Method is explained in [11]. An efficient video compression technique as modified HEVC coding based on saliency features to counter 
the existing drawbacks is introduced in [12]. DWT Based Image Compression using Frequency Band Suppression and Throughput Enhancement is described in [13]. Comparative Study of Wavelet Image Compression: JPEG2000 Standart using DWT and lead to an image data compression while preserving the essential quality of the original image is discussed in [14].

\subsection{The Problem}

The main drawback is that users cannot control the image quality over the background region or between regions with different degree of interest. For solving network bandwidth, security problems and storage problems, image need to be compressed.

\section{Proposed Solution}

Our proposed system is a method of lossy based image compression scheme based on the EZW algorithm. In this method the compression is done by extracting the ROI regions from the original satellite images. Then the compression algorithm of EZW algorithm is applied and is compressed. The construction of the system is shown in Figure 1.

\subsection{Extraction of ROI}

The ROI extraction is the first step that is carried out in our proposed system. This ROI image is the images that are used for the compression process so as to increase our compression accuracy. The system is said to be as the lossy compression method. The reason for this is due to the usage of the ROI image for the compression process. From the ROI images the lossy compression is done by using the EZW algorithm.

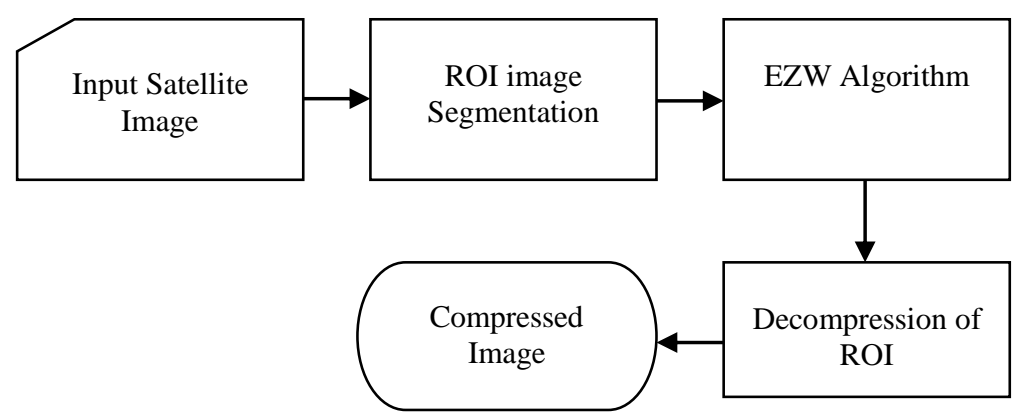

Figure 1. Framework of the proposed image compression system

\subsection{EZW Compression Algorithm}

The EZW algorithm is the one which is used for the compression process. Here the ROI images extracted from the original images are taken as the input for the EZW algorithm that is used for the compression. This algorithm is based on the DWT wavelet concept. By the consideration of the transformed coefficients as a tree where the coefficients of lower frequency at the root node and the spatially related coefficients present in the higher frequency of one or more sub-trees, which are zero or nearly zero are called as zero-trees.

\section{Results and Discussion}

The image compression technique based on the ROI image based EZW algorithm is said to be as the lossy compression technique in which the compression occurs without any dataloss in it so that only the images are compressed without loss of data. The output result is as shown in Figure 2. 


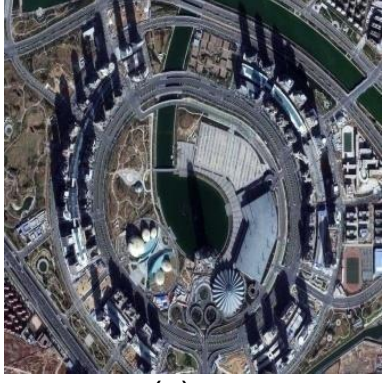

(a)

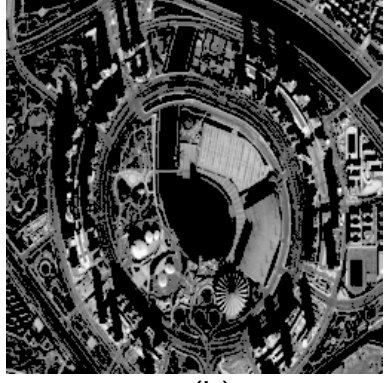

(b)

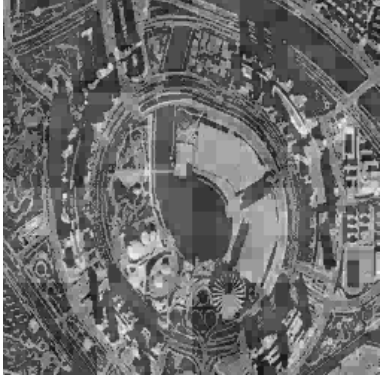

(c)

Figure 2 (a) Original image (b) Extracted ROI (c) Compressed output image

\section{Conclusion}

The proposed image compression method based on the ROI extracted based lossy compression method by using the EZW algorithm is discussed. The method is tested by the literally available satellite images. And the performance of the proposed system is analyzed by calculating the PSNR values of the compressed image. Our proposed system has obtained PSNR values of about $90.6 \% \mathrm{db}$.

\section{References}

[1] Doukas C, Maglogiannis I. Region of interest coding techniques for medical image compression. IEEE Engineering in medicine and Biology Magazine. 2017; 26(5): 29-35.

[2] Perumal B, Rajasekaran M P. A hybrid discrete wavelet transform with neural network back propagation approach for efficient medical image compression. IEEE International Conference on Emerging Trends in Engineering, Technology and Science. 2017; 1-5.

[3] Sharma S, Bhat U. Image Compression using an efficient hybrid algorithm, 2013.

[4] Wu Y G. Medical image compression by sampling DCT coefficients. IEEE Transactions on Information Technology in Biomedicine. 2002; 6(1): 86-94.

[5] Wu Y G, Tai S C. Medical image compression by discrete cosine transforms spectral similarity strategy. IEEE Transactions on Information Technology in Biomedicine. 2001; 5(3): 236-243.

[6] Reddy B V, Reddy P B, Kumar P S, Reddy A S. Lossless Compression of Medical Images for Better Diagnosis. IEEE 6thInternational Conference on Advanced Computing. 2016; 404-408.

[7] Savitri P A I, Murdiansyah D T, Astuti W. Digital medical image compression algorithm using adaptive Huffman coding and graph based quantization based on IWT-SVD. IEEE 4thInternational Conference on Information and Communication Technology. 2016; 1-5.

[8] Bin L, Qinggang M. An improved SPIHT wavelet transform in the underwater acoustic image compression. IEEE International Conference on Measurement, Information and Control. 2013; 2: 1315-1318.

[9] Panda S S, Jena, G. Image Super Resolution Using Wavelet Transformation Based Genetic Algorithm. Springer India Computational Intelligence in Data Mining. 2016; 2: 355-361.

[10] Panda S S, Jena G, Sahu S K. Image super resolution reconstruction using iterative adaptive regularization method and genetic algorithm. Springer India computational Intelligence in Data Mining. 2015; 2: 675-681.

[11] Saniei R, Faez K. The Security of Arithmetic Compression Based Text Steganography Method. International Journal of Electrical and Computer Engineering. 2013; 3(6): 797.

[12] Rekha B. High Definition Video Compression Using Saliency Features. Indonesian Journal of Electrical Engineering and Computer Science. 2017; 7(3).

[13] Lakshmi Narayanan K, Ramesh G P. Discrete Wavelet Transform Based Image Compression using Frequency Band Suppression and Throughput Enhancement. International Journal of MC Square Scientific Research. 2017; 9(2): 176-182.

[14] Ennaciri A, Erritali M, Mabrouki M, Bengourram J. Comparative Study of Wavelet Image Compression: JPEG2000 Standart. Indonesian Journal of Electrical Engineering and Computer Science. 2015; 16(1): 83-90. 